\title{
Statistical analysis of scattered field by building facades
}

\section{Other Conference Item}

\section{Author(s):}

Kersaudy, P.; Mostarshedi, S.; Sudret, Bruno (D); Picon, O.; Wiart, J.

Publication date:

2015

Permanent link:

https://doi.org/10.3929/ethz-a-010582934

Rights / license:

In Copyright - Non-Commercial Use Permitted

Originally published in:

https://doi.org/10.1109/URSI-AT-RASC.2015.7303225 


\title{
Statistical Analysis of Scattered Field by Building Facades
}

\author{
P. Kersaudy ${ }^{(1)}$, S. Mostarshedi ${ }^{(2)}$, B. Sudret ${ }^{(3)}$, O. Picon ${ }^{(2)}$, and J. Wiart ${ }^{(1)}$
}

(1) Orange Labs, 80305 Issy-les-Moulineaux, France

(2) ESYCOM (EA 2552), Université Paris-Est, 77524 Marne-la-Vallée, France

(3) Institute of Structural Mechanics, ETH, CH-8093 Zurich, Swiss

Wireless communications experienced a great expansion worldwide and the networks are very dense in urban areas. Predictive tools are essential to assess the distribution of electromagnetic fields in a fast and accurate way in order to optimize the implementation of base stations and minimize the human exposure. Wave propagation simulators are based on different models of urban environment and employ appropriate calculation methods. We can mention empirical, statistical, theoretical, site-specific models or a combination of them to generate a hybrid model. Given the complex and variable environment, the prediction of electromagnetic fields with such deterministic simulators has to be accompanied by an acceptable amount of uncertainty. Consequently the assessment of the electromagnetic waves in urban environment in terms of the propagation of uncertainties presents an important challenge. In order to integrate the statistical dimension into simulations and measurements, different numerical methods can be used. Here we propose the polynomial chaos expansion which is a good candidate for a parsimonious approach able to provide with various types of statistical information. This method is an advanced statistical technique which by using a polynomial expansion, provides a meta-model for the physical model.

The meeting presentation will concentrate on the statistical assessment of electromagnetic field which is as follows. A 2D model for building facade including different architectural details has been adopted. The computational method is based on the Green's functions associated with the interface between two semi infinite media. Among all possible sources of uncertainty, 8 random variables have been defined to represent the random variation of some geometrical and electrical parameters of the building facade. Two observation scenarios have been defined, one in specular direction where the normally incident plane wave illuminates the random building facade. The second is defined in non-specular reflection where the observation is made for the angles other than the normal direction. Both scenarios can be tested in different diffraction zones of the building going from the near-field to the far-field. For each scenario, the polynomials constituting the basis of the probabilistic space are chosen according to the input random variables distributions. The expansion is then truncated using Least Regression Angle (LARS) in order to obtain a sparse representation of the physical model. By minimizing the mean square error between the actual physical model computed at a given point of the experimental design and the truncated polynomial approximation, the unknown coefficients can be calculated. The quality of the meta-model is assessed using a Leave-One-Out technique and the points in the experimental design are iteratively adjusted according to the desired model quality and using a Nested Latin Hypercube Sampling. Finally using the best available meta-model, the global sensitivity indices are calculated for each of the input parameters and the statistical distributions of the field at desired distances are presented.

One of the direct results of this study is to simplify the complex mechanism between the input and output of a system by underlining the most influent parameters in a given configuration. In a complex computational system such as field scattering evaluation in urban area with a large number of input random variables, this allows a smart elimination of the less influent parameters and thus a simplification of the whole process. 